Now there is an undoubted analogy between this and the experiments under discussion. The medium containing resonators is in this case the layer immediately above the earth's surface containing the tuned aerials. We must, therefore, take into account the possible effect of a change in the refractive index of this medium as well as in its absorption factor. But the theory of wireless wave propagation as developed by Sommerfeld and others shows that the former factor may be as important as the latter in determining the attenuation which the waves will experience in passing over the earth's surface. Finally, the theory also shows that a variation of the refractive index with the wave-length in the manner demonstrated in theoretical and experimental optics in such circumstances (anomalous dispersion) would have a tendency to cause the point of maximum attenuation (and therefore minimum received intensity) to be shifted to the left of the critical wave-length line, as it was found to be from observation. This is manifestly only a tentative explanation, but it is enough to show that this unexpected feature in the experimental results does not invalidate the general conclusion that the large number of receiving aerials in the London area have a very marked absorbing effect on the waves passing over them.

The observations on which the above note is based were carried out as part of an investigation for the Radio Research Board of the Department of Scientific and Industrial Research.

R. H. BARFIFLD

Radio Research Station,

Ditton Park, Slough, Bucks, Dec. 6.

\section{Relation between the Reciprocal Impenetrability of Matter and Pauli's Exclusion Principle.}

WHEN the collisions between the molecules of an ideal gas are taken into consideration, then the motion of the gas does not appear to be multiply periodic. It is therefore impossible to quantise this motion by means of the 'classical' quantum theory. Moreover, in the treatment of the ideal gas by means of the new ' undulatory mechanics,' hitherto only the impenetrability of the walls of the vessel has been taken into account, and not the reciprocal impenetrability of the molecules (E. Schrödinger, Phys. Zs., 27, 95, 1926). I propose to show here that, in the case of impenetrable moving mass-points, wavemechanics gives an exact and very elementary solution. Simultaneously we arrive at the relation mentioned in the title.

For purposes of brevity we will limit ourselves to a 'one-dimensional' gas : $N$ monatomic mass-points of equal mass $M$ are enclosed in a tube, which extends from $x=-\frac{\pi}{2}$ to $x=+\frac{\pi}{2}$. If we first take into account only the impenetrability of the ends of the tube, then we have to look for all the solutions of the $N$-dimensional Schrödinger-equation :

$$
\left[\frac{\partial^{2}}{\partial x_{1}{ }^{2}}+\frac{\partial^{2}}{\partial x_{2}{ }^{2}}+\ldots+\frac{\partial^{2}}{\partial x_{N}{ }^{2}}+\frac{8 \pi^{2} M}{h^{2}} E\right] \psi=0,
$$

which are equal to zero at the faces of the $N$-dimensional $\pm \frac{\pi}{2}$-cube. These solutions are :

$$
\psi=\cos a_{1} x_{1} \cos a_{2} x_{2} \ldots \cos a_{N} x_{N},
$$

where $a_{1} \ldots \alpha_{N}$ are arbitrary positive integers and where $E=\frac{h^{2}}{8 \pi^{2} M}\left(a_{1}^{2}+\ldots a_{N}^{2}\right)$. To the same $E$, and with the same numbers $a_{1} \ldots a_{N}$, there belong also all the solutions obtained from solution (2) by ex- changing all the co-ordinates $x_{1} \ldots x_{N}$ with each other, at the same time keeping the numbers $a_{1} \ldots a_{N}$ fixed. By multiplication of all these solutions by arbitrary constants and addition, we obtain the most general solution of this kind :

$\Psi=\Sigma C\left(p_{1} \ldots p_{N}\right) \cos \alpha_{1} x_{p_{1}} \cos a_{2} x_{p_{2}} \ldots \cos \alpha_{N} x_{p_{N}}$,

where $p_{1} \ldots p_{N}$ signifies one permutation of the numbers $1 \ldots N$, and the summation must be taken over all the permutations.

Now let us also consider the reciprocal impenetrability of the molecules. That can be done by means of the additional 'diagonal-restriction'; which in wave-mechanics corresponds to the impossibility of two molecules occupying the same point in space at the same time. Of all the solutions of the form (3), only those are allowed which are also equal to zero at all points of the $\frac{N(N-1)}{2}$ flat 'diagonal-spaces' of $N-1$ dimensions which are represented by the equations $x_{h}=x_{k}(h, k=1, \ldots N)$. It can be shown now best by considering first the cases $N=2$ and $N=3$ that all the constants $C\left(p_{1} \ldots p_{N}\right)$ must have values equal to each other or opposite in sign, according as the arguments $p_{1} \ldots p_{N}$ are derived from the numbers $1 \ldots N$ by an even or an odd number of simple permutations. Or, in other words, because of the reciprocal impenetrability of the molecules only the antisymmetric solutions are allowed :

$$
\Psi^{\text {antisym }}\left(x_{1} \ldots x_{N}\right)=C \cdot\left|\begin{array}{cc}
\cos a_{1} x_{1} \ldots & \cos a_{1} x_{N} \\
\vdots & \vdots \\
\vdots & \vdots \\
\cos a_{N} x_{1} \ldots & \cos a_{N} x_{N}
\end{array}\right| .
$$

Thus, just those solutions which, according to the researches of Heisenberg and Dirac, are connected with Pauli's exclusion principle (the exclusion of equal quantum numbers for two electrons in the same atom-in our case for two gas molecules in the same vessel), the determinant becomes identically equal to zero when two of the numbers $\alpha_{s}$ are equal to each other.

Finally, we may remark as follows :

I. For a three-dimensional gas contained in a vessel of general form, naturally $\psi_{\alpha \beta \gamma}(x y z)$ must be substituted for $\sin a x$; further, in the 'diagonal-restriction' the equations $\left(x_{h}-x_{k}\right)^{2}+\left(y_{h}-y_{k}\right)^{2}+\left(z_{h}-z_{k}\right)^{2}=0$ for $x_{h}=x_{k}$, and we have always to exchange a triplet $x_{h} y_{h} z_{h}$ with a triplet $x_{k} y_{k} z_{R}$.

II. In order to show that the impenetrability of the walls and for the molecules must really be expressed by the corresponding zero-conditions of the solutions of the Schrödinger equation, we approximate first this impenetrability by continuously varying forces of repulsion and then we go in the resulting solutions to the limit.

III. At the same time we get very simple models for the treatment of the question of how far the Pauli's exclusion principle holds for two electrons if they are in the same atom but not if they belong to distinotly separated atoms.

IV. The non-validity of Pauli's principle for ' lightcorpuscles' should now perhaps be connected with their reciprocal penetrability, though the very provisional and symbolical character on one hand of the light corpuscles, and on the other of the multidimensional wave-equation (1), should specially be remembered here. These remarks and some connected statistical questions will be dealt with elsewhere.

\section{Paul Ehrenfest.}

Instit. v. theor. Natuurkunde, Leiden, Holland.

No. 2988, VoL. 119] 